\title{
Penile Angiokeratomas (PEAKERs) Revisited: A Comprehensive Review
}

Philip R. Cohen · Nicholas J. Celano

Received: March 31, 2020 / Published online: June 6, 2020

(C) The Author(s) 2020 usually bleeding and increasing size and less often abrupt onset, pain and pruritus. The glans penis $(55.5 \%)$ and the penile shaft (35\%) were the most common sites of PEAKERs; the angiokeratomas were also located on the foreskin $(5.5 \%)$ or both the glans penis and penile shaft (4\%). Thirty seven percent of patients with glans penis PEAKERs only had angiokeratomas on the corona. Scrotal angiokeratomas were also present in $20 \%$ of patients with PEAKERs. A solitary PEAKER was observed in $32 \%$ of the men. Most of the PEAKERs were $1-5 \mathrm{~mm}$ in size. The PEAKERs presented as purple, red and/or blue papules; 70\% of the men's PEAKERs were more than one color. Clinical features often established the diagnosis; in addition, some of the men's angiokeratomas were biopsied or evaluated with dermoscopy. Laser therapy, in $56 \%$ of the men, was the most common treatment modality. Less common interventions included electrocautery, radiofrequency and excision. PEAKER recurrence or persistence was observed after excision (two men) or cryotherapy (one man), respectively. Several of the men (27\%) decided to observe their PEAKERs without treatment.

Keywords: Angiokeratoma; Clitoris; Genital; PEAKER; Penile; Penis; Rejuvenation; Scrotal; Scrotum; Vulva

P. R. Cohen - N. J. Celano

Touro University California College of Osteopathic

Medicine, Vallejo, CA, USA 


\section{Key Summary Points}

Penile angiokeratomas (PEAKERs) is a subtype of genital angiokeratoma in men.

Bleeding and increasing size were the most common PEAKER-associated symptoms.

PEAKERs were usually located on the glans penis and penile shaft; one-fifth of the men also had scrotal angiokeratomas.

Clinical features (a purple, red and/or blue, 1-5 $\mathrm{mm}$, papule) were often used to establish the diagnosis of a PEAKER.

Laser therapy was the most common modality used to treat PEAKERs.

\section{INTRODUCTION}

Angiokeratomas are benign vascular lesions characterized by overlying hyperkeratosis and dilated superficial blood vessels. In February 1967, a classification of angiokeratoma was proposed by Imperial and Helwig [1]. The five clinical types of angiokeratoma include angiokeratoma corporis diffusum (Fabry's disease), angiokeratoma of Mibelli, angiokeratoma of Fordyce, angiokeratoma circumscriptum, and solitary or multiple angiokeratoma [1].

Fordyce angiokeratoma, also referred to as genital angiokeratoma, was initially described by John Addison Fordyce in 1896 [2]. Scrotal angiokeratoma in men is analogous to angiokeratoma of the vulva in women [3]. Recently, a subtype of genital angiokeratoma in men-penile angiokeratoma (PEAKER)-has been introduced [4].

The PubMed database was used to search the following words: angiokeratoma, clitoris, genital, peaker, penile, penis, rejuvenation, scrotal, scrotum and vulva. The relevant papers and references cited in those papers that were generated by the search were reviewed; PEAKERs have been described in 54 men. The purpose of this article is to summarize the features of PEAKERs (Table 1) [1, 4-25].

Although angiokeratomas are typically located in the bathing trunk area (and particularly on the penis and scrotum) of men with Fabry's disease, these individuals are not included in the men with PEAKERs discussed in this paper [26-30]. This article is based on previously conducted studies and does not contain studies with human participants or animals performed by any of the authors. However, the patients or their parent signed a consent providing permission for including the clinical photographs in this article.

\section{DISCUSSION}

\section{History}

The original report of a genital angiokeratoma was described in a 60-year-old man with urinary trouble who was admitted to a New York City hospital. Dr. Fordyce noticed many small, spherically shaped, dark purple lesions on his scrotum. A skin biopsy from one of the lesions demonstrated an angiokeratoma; Fordyce differentiated these angiokeratomas from those reported by Mibelli based on the following: his patient was older (instead of being a child or an adolescent), the lesions were located on the scrotum (instead of the hands and feet), and there was no history of chilblains [2, 31].

The vulva is the embryologic analog of the scrotum. Angiokeratoma of the vulva (labia majora) was initially described in a 58-year-old woman; the lesions had been present for 2 years, bilateral in distribution, verrucous in appearance and occasionally bleeding [32]. Since then, multiple additional women with vulvar angiokeratoma have been reported $[3,7,12,13,15,33-37]$.

To the best of our knowledge, angiokeratoma of the penis-a subtype of genital angiokeratoma-was reported by Imperial and Helwig in their study on angiokeratoma in 1967. Thirteen men had a solitary angiokeratoma on either the prepuce (foreskin, three men) or shaft (ten men) of the penis. None of the men has 
Table 1 Characteristics of 54 patients with penile angiokeratomas (PEAKERs) ${ }^{\mathrm{a}, \mathrm{b}}$

\begin{tabular}{|c|c|c|c|c|c|c|c|c|c|c|c|c|}
\hline C & $A^{c}$ & Dur & NoA & Sze & Color & GP & PS & $S$ & $S_{x}$ & $\mathbf{B x}$ & Treatment & Ref. \\
\hline 1 & $<1$ & $0^{\mathrm{d}}$ & 2 & NS & $\operatorname{Pr}$ & $+\mathrm{m}$ & - & - & + & + & Excision; PDL & 5 \\
\hline 2 & $<10$ & NS & Mny & VS & $\operatorname{Pr}$ & + & - & + & - & + & None & 6 \\
\hline 3 & $<10$ & $20+$ & $>30$ & NS & NS & - & + & + & + & - & Nd:YAG & 7 \\
\hline 4 & 10 & 27 & Mul & 2 to 4 & BlRd & $+c$ & - & - & + & + & Nd:YAG ${ }^{\mathrm{e}}$ & 8 \\
\hline 5 & 11 & 5 & 3 & 1 to 2 & $\operatorname{Pr}$ & $+\mathrm{m}$ & - & - & + & - & EC & CR1 \\
\hline 6 & 14 & $\mathrm{AC}$ & Mul & 2 to 4 & PrRd & + & - & - & - & + & None & 9 \\
\hline 7 & 16 & 5 & Mny & NS & NS & - & + & + & - & - & $\mathrm{EC}$ & 10 \\
\hline 8 & 19 & 0.5 & NS & 1 to 5 & BlRd & $+c^{f}$ & - & - & + & + & None & 11 \\
\hline 9 & 23 & 3 & F-S & 1 to 5 & BlPrRd & + & - & - & - & $\mathrm{D}$ & PDL/Nd:YAG & $12 \mathrm{C} 1$ \\
\hline 10 & 24 & 1 & $>10$ & NS & NS & - & + & - & - & $\mathrm{D}$ & $595 \mathrm{VPPDL}$ & $13 \mathrm{C} 24$ \\
\hline 11 & 25 & 1 & Mul & 2 to 4 & $\mathrm{Bk}$ & $+c$ & - & - & - & + & $\mathrm{EC}$ & 14 \\
\hline 12 & 26 & 5 & F-S & 1 to 5 & BlPrRd & - & + & - & - & $\mathrm{D}$ & PDL/Nd:YAG & $12 \mathrm{C} 9$ \\
\hline 13 & 26 & 5 & F-S & 1 to 5 & BlPrRd & + & - & - & - & $\mathrm{D}$ & PDL/Nd:YAG & $12 \mathrm{C} 15$ \\
\hline 14 & 26 & 2 & $>10$ & NS & NS & + & + & - & - & $\mathrm{D}$ & VPPDL & $13 \mathrm{C} 1$ \\
\hline 15 & 26 & 2 & NS & NS & NS & + & - & - & - & $\mathrm{D}$ & VPPDL & $13 \mathrm{C} 3$ \\
\hline 16 & 28 & 7 & NS & NS & NS & + & - & - & - & $\mathrm{D}$ & VPPDL & $13 \mathrm{C} 2$ \\
\hline 17 & 28 & 1 & NS & NS & NS & + & - & - & - & $\mathrm{D}$ & VPPDL & $13 \mathrm{C} 8$ \\
\hline 18 & 29 & 6 & F-S & 1 to 5 & BlPrRd & + & - & - & - & $\mathrm{D}$ & PDL/Nd:YAG & $12 \mathrm{C} 4$ \\
\hline 19 & 30 & 13 & NS & 1 to 8 & BlPrRd & - & + & + & - & - & Nd:YAG & $15 \mathrm{C} 9$ \\
\hline 20 & 31 & 2 & F-S & 1 to 5 & BlPrRd & - & + & - & - & $\mathrm{D}$ & PDL/Nd:YAG & $12 \mathrm{C} 14$ \\
\hline 21 & 32 & 27 & NS & 1 to 8 & BlPrRd & - & + & - & - & - & Nd:YAG & $15 \mathrm{C} 10$ \\
\hline 22 & 33 & 30 & 1 & 2 by 2 & $\operatorname{Pr}$ & $+c$ & - & - & - & - & None & 4 \\
\hline 23 & 33 & 1 & 3 & 3 to 9 & BkTan & - & + & - & - & - & Excision & 16 \\
\hline 24 & 40 & 21 & $>5$ & 1 to 8 & BlPrRd & + & - & - & - & - & Nd:YAG & $15 \mathrm{C} 3$ \\
\hline 25 & 41 & 8 & NS & NS & NS & + & + & - & - & $\mathrm{D}$ & VPPDL & $13 \mathrm{C} 12$ \\
\hline 26 & 42 & 0.67 & Mul & 0.5 to 3 & $\mathrm{BlPr}$ & + & - & - & + & + & None & 17 \\
\hline 27 & 43 & NS & Num & NS & PrRd & $+c^{f}$ & - & - & - & + & None & $18 \mathrm{C} 2$ \\
\hline 28 & 52 & 0.17 & $>10$ & NS & BlRd & $+c$ & - & - & - & + & Erb:YAG/KTP & 19 \\
\hline 29 & 56 & 2 & 1 & 1 by 2 & $\mathrm{BkPr}$ & + & - & - & - & + & Cryo; Excision & 20 \\
\hline 30 & 59 & 8 & $>30$ & 1 to 3 & $\operatorname{Pr}$ & $+c$ & - & + & + & - & None & CR2 \\
\hline 31 & 62 & 0.5 & $>30$ & 0.5 to 2 & BlPrRd & $+\mathrm{c}$ & - & - & + & + & None & 21 \\
\hline 32 & 62 & Yrs & 3 & NS & $\operatorname{Pr}$ & $+c$ & - & - & - & - & None & 22 \\
\hline
\end{tabular}


Table 1 continued

\begin{tabular}{lllllllllllll}
\hline $\mathbf{C}$ & $\mathbf{A}^{\mathbf{c}}$ & Dur & NoA & Sze & Color & GP & PS & S & Sx & Bx & Treatment & Ref. \\
\hline 33 & 66 & NS & Mul & NS & PrRd & + & - & + & - & - & None & $18 \mathrm{C} 1$ \\
34 & 68 & 4 & Mul & 10 to 15 & NS & - & + & + & + & + & Rfx; EC & 23 \\
35 & 71 & 0.25 & 5 & $<4$ & PrRd & + & - & - & - & + & Emollients & 24 \\
36 & 75 & NK & 7 & 1 to 3 & Pr & $+\mathrm{c}^{\mathrm{f}}$ & - & + & + & - & None & CR3 \\
\hline
\end{tabular}

A (age in years), $A C$ after circumcision, $B k$ black, $B l$ blue, $B X$ biopsy (was used to make the diagnosis of angiokeratoma), $C$ case, $c$ corona, $C R$ current report, $C r y o$ cryosurgery with liquid nitrogen, $D$ dermoscopy (was used to make the diagnosis of angiokeratoma), Dur duration (number of years angiokeratomas were present prior to establishing the diagnosis, $E C$ electrocautery, Erb:YAG 2940-nm erbium: yttrium aluminium garnet, $F-S$ few to several, GP glans penis, KTP 532-nm potassium-titanyl-phosphate, $m$ meatus, $M n y$ many, $M u l$ multiple, $N o A$ number of angiokeratomas, Nd:YAG 1064-nm neodymium-doped: yttrium aluminum garnet, $N K$ not known, $N S$ not stated, $N u m$ numerous, $P D L 595-\mathrm{nm}$ pulsed dye laser, $\operatorname{Pr}$ purple, $P S$ penile shaft, $R d$ red, $R e f$ reference, $R f x$ radiofrequency surgical excision (for larger lesions), $S$ scrotum, $S x$ symptoms, Sze size (in millimeters), VPPDL 595-nm variable-pulsed pulsed dye laser, VS very small, Yrs years, + present, absent, $<$ less than, $>$, greater than

${ }^{a}$ Cases 37-41 are 5 of 12 men included in a study of angiokeratoma of Fordyce treatment with $585-\mathrm{nm}$ pulsed dye laser. The men ranged from 21 to 76 years of age at the time of treatment; the angiokeratomas had been present for 0.5 to 3 years. The number of angiokeratomas ranged from few to several dozen; they were 2-7 $\mathrm{mm}$ in size. A photograph shows an angiokeratoma on the corona of the glans penis (even though the Subjects and methods section describes the lesion to be on the penile shaft); therefore, based on the image, all five men had angiokeratomas on their glans penis (and one definitely had the lesion on his corona); three of the men also had lesions on their scrotum. The angiokeratomas were symptomatic: they had increased in number, they spontaneously bled, and they were a source of social embarrassment. All of the lesions were treated with 585-nm pulsed dye laser for two to six treatments every 2 months [25]

${ }^{\text {b }}$ Cases 42 to 54 are $13(14 \%)$ of the 95 men with either solitary or multiple angiokeratomas. The patients were included in a retrospective study of 116 individuals (of which $82 \%$ were men) with angiokeratomas collected from the files from the Armed Forces Institute of Pathology during the period from 1942 through 1963. The solitary lesions were located on the penile shaft: on either the prepuce (foreskin, in three men) or the penis (in ten men) [1]

${ }^{c}$ This is the age when the initial appearance of the angiokeratoma was noticed by the patient (or their family); if the patient was unaware of the angiokeratoma, it is the age that the angiokeratoma was described by the patient's clinician

$\mathrm{d}$ The patient's original glans penis angiokeratoma was congenital and removed surgically during infancy. At age 7 years, he developed a recurrent biopsy-confirmed angiokeratoma at the same site. The recurrent PEAKER had been present for 6 months prior to evaluation; it was successfully treated, without recurrence, at 1-year follow-up, with three treatments using a 595-nm pulsed dye laser.,

e The laser used was a 532-nm neodymium-doped: yttrium aluminum garnet

${ }^{f}$ Angiokeratomas were present on both the corona and the glans penis

angiokeratoma on either the glans penis or the scrotum [1].

Angiokeratomas of the glans penis are not commonly described. Indeed, a review of glans penis angiokeratoma in 2017 only identified 12 men [20]. Subsequently, a year later, a paper on penile angiokeratoma added another patient with an angiokeratoma of the glans penis to the 20 previously reported men [4]; the paper included 12 men from Beutler and Cohen's [2] review and additional patients from two subsequent studies: three men from Ibrahim's [12] investigation and five men from Baumgartner and Simaljakova's [13] study.

\section{Nomenclature}

Fordyce angiokeratoma was initially reported as lesions on the scrotum [2]. However, this 
clinical variant now includes angiokeratomas of both the male and female genitalia-not only the scrotum, but also the vulva. However, genital angiokeratoma also includes angiokeratoma of the penis and angiokeratoma of the clitoris.

Basu and Cohen, in a review of angiokeratoma of the glans penis, introduced the acronym PEAKER for penile angiokeratoma [4]. The 'PE' refers to the first two letters of 'penile,' the 'A' refers to the first letter of 'angiokeratoma,' and the 'KER' refers to the sixth, seventh and eighth letters of 'angiokeratoma.' The previous paper on PEAKERs only included men with angiokeratomas on the glans penis [4]; however, the current paper appropriately expands the concept of PEAKERs to include angiokeratomas not only of the glans penis, but also of the penile shaft and foreskin.

The clitoris is the embryologic analog of the penis. However, in contrast to angiokeratoma of the penis, reports of clitoral angiokeratoma are rare [38-40]. We introduce the acronym 'CLANKER' for clitoral angiokeratoma; the ' $\mathrm{CL}$ ' refers to the first two letters of 'clitoral,' the ' $\mathrm{AN}^{\prime}$ refers to the first two letters of 'angiokeratoma,' and the 'KER' refers to the sixth, seventh and eighth letters of 'angiokeratoma.'

\section{Occurrence}

The prevalence of genital angiokeratomas remains to be definitively established. Imperial and Helwig performed a retrospective study of the 116 angiokeratoma pathology specimens from the files of the Armed Forces Institute of Pathology that were collected from 1942 to 1963. Eighty-two percent (95 of 116) of the patients were men. Genital angiokeratomas were only observed in 13 men; they were located on either the penis ( 10 men) or the foreskin (3 men). Hence, in this study, the prevalence of genital angiokeratomas in a population of men with angiokeratomas was almost $14 \%$ (13 of 95) [1].

Another retrospective study was performed by Spanish investigators. The researchers evaluated the histologic and ultrastructural features of 93 biopsy-confirmed angiokeratomas that were observed during a 30-year period (from
1955 to 1985) in the Department of Medicine and Surgery of the School of Medicine of the University of Granada. The prevalence of genital angiokeratomas was $14 \%$ (13 of 93 cases) [41].

Subsequently, $>50$ years after Imperial and Helwig's study, Sadowsky et al. were prompted to determine the prevalence of genital angiokeratoma in adults. Between 23 November 2013 and 16 October 2014, a senior dermatologist in the outpatient Dermatology Department at Rush University Medical Center in Chicago, IL, examined the genitalia of 213 white European American adults during melanoma screening and surveillance. Genital angiokeratomas were observed in $37 \%$ ( 47 of 127 ) of the men and $20 \%$ (17 of 86$)$ of the women [34].

Three investigators have evaluated the prevalence of genital angiokeratomas in men prior to and following the age of 49,50 or 51 years [34, 42, 43]. However, all of these researchers did not account for the duration of the lesions when they recorded the onset age for the appearance of the angiokeratomas in these men. Sandowsky et al. observed more than a two-fold greater prevalence of genital angiokeratomas in men $\geq 51$ years of age $(48 \%, 39$ of 81 men) versus men $\leq 50$ years of age $(17 \%, 8$ of 46 men) [34].

Izaki recorded the prevalence of scrotal angiokeratomas in Japanese men. However, Izaki noted a much lower prevalences of genital angiokeratoma than that described by Sandowsky et al. Specifically, Izaki only observed a prevalence of $15 \%$ (23 of 154 men) scrotal angiokeratomas in men $\geq 51$ years of age compared with $2 \%$ (27 of 1398 men) $\leq 50$ years of age [42].

Bean evaluated the prevalence of angiokeratoma of the scrotum in men $\geq 50$ years [43]. When Sadowsky et al.'s data were analyzed with these parameters, they observed genital angiokeratomas in $48 \%$ (40 or 83 ) of men > 49 years years old [34]. In contrast, Bean-similar to Izaki-only observed the prevalence of scrotal angiokeratomas to be $14 \%$ (70 of 500 men) [43].

The incidence of PEAKERs has not previously been determined. Several studies evaluating the characteristics and/or treatment of genital 
angiokeratomas have included patients with either angiokeratomas of the scrotum or penis or both (Table 2) $[10,12,13,15,25]$. The incidence of PEAKERs in these investigations ranged from 3\% ( 1 of 35 men) to $42 \%$ (5 of 12 men). When the data from these studies are combined, the incidence of PEAKERs in men with genital angiokeratomas is 22\% (20 of 93 men). Whether this calculated incidence of penile angiokeratomas accurately reflects the occurrence of PEAKERs in men with genital angiokeratomas requires additional observations.

\section{Epidemiology}

To the best of our knowledge (and including the three patients presented in the legends of Figs. 1, 2, 3 in this article), PEAKERs have been described in 54 men (Table 1) [1, 4-25]. The exact onset age-when the angiokeratoma was initially observed by the patient or their parents-was available for 36 of the patients. The onset age of PEAKERs ranged from $<1$ year of age to 75 years old (median, 30 years old).

Congenital genital angiokeratomas are uncommon [44]. However, the youngest patient with a PEAKER was a 7-year-old boy with a recurrent lesion on his glans penis of 6-month duration that had increased in size and frequently bled. However, the boy had had a similar lesion that was present at birth and had been surgically removed when he was an infant [5].

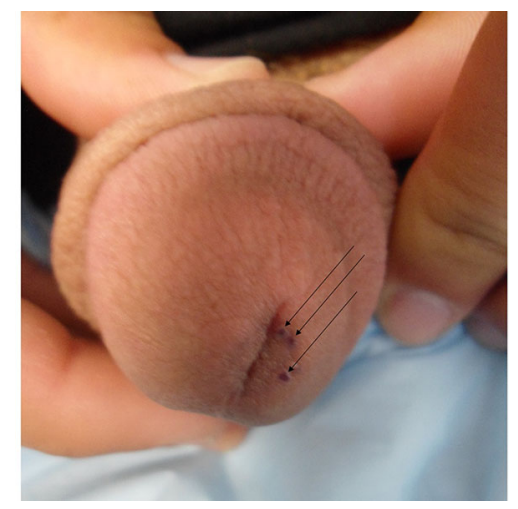

Fig. 1 Penile angiokeratomas (PEAKERs) on the glans penis (urethral meatus) of a 16-year-old man. The PEAKERs were initially noticed 5 years ago, when he was age 11 years. He came for an evaluation since he was concerned that the lesions were warts. They had increased in number and had become more noticeable. Occasionally, they bled when they came in contact with his clothing. Examination showed a circumcised man with three 1-2 $\mathrm{mm}$ purple papules on the left side of his glans penis adjacent to the urethral meatus (black arrows). The diagnosis of angiokeratoma was established based on the clinical appearance of the lesions. The patient was concerned because the lesions periodically bled. Therefore, the PEAKERs were treated with electrocautery. Topical anesthetic (using a cream containing an equal mixture of lidocaine $2.5 \%$ and prilocaine $2.5 \%$ ) was applied to the glans penis for an hour. The hyfrecator was set a 5 , and each of the lesions received a single electrocautery application of $<1 \mathrm{~s}$. All three of the lesions completely resolved; there has been no recurrence at 8 months following the treatment session

Table 2 Incidence of penile angiokeratomas (PEAKERs) in men the genital angiokeratomas

\begin{tabular}{lcccc}
\hline Author (Year of publication) & PEAKERs & Men with GA & Percent & Ref. \\
\hline Imperial \& Helwig (1967) & 1 & 35 & 3 & {$[10]$} \\
Baumgartner \& Simaljakova (2017) & 6 & 22 & 27 & {$[13]$} \\
Ibrahim (2015) & 5 & 16 & 31 & {$[12]$} \\
Ozdemir et al. (2008) & 3 & 8 & 38 & {$[15]$} \\
Lapidoth et al. (2006) & 5 & 12 & 42 & {$[25]$} \\
Total & 20 & 93 & 22 & \\
\hline
\end{tabular}

GA genital angiokeratomas, PEAKERs penile angiokeratomas, Ref references 


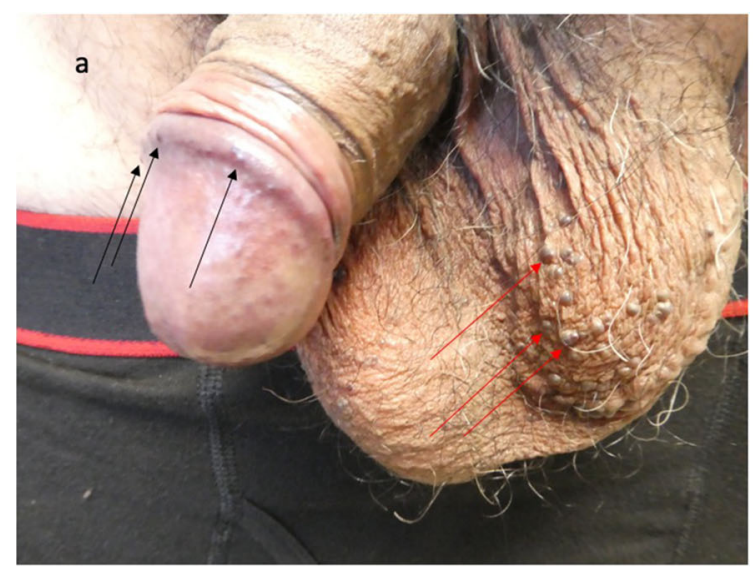

Fig. 2 A 67-year-old man with penile angiokeratomas (PEAKERs) on the corona of the glans penis and scrotal angiokeratomas. He presented for an evaluation of the vascular lesions on his scrotum, which had been present for 8 years and would occasionally bleed; he was unaware of the similar-appearing lesions on his glans penis. In addition, his history was remarkable for previously having redness on his distal penis that resolved with a whitening of the area. Cutaneous examination of his uncircumcised penis and scrotum was performed. Distant (a) and closer

The age range between 20-29 years was the most frequent for the onset of PEAKERS [10 of 36 men (28\%)]; this observation is similar to that of Imperial and Helwig who noted that scrotal angiokeratomas most commonly appeared in men during their 2nd decade: 12 of 35 men (34\%) [10]. PEAKERs appearing in children ( $<18$ years of age) account for $17 \%$ (7 of 41) of these angiokeratomas. Indeed, only half of the patients ( 18 of 36 men) were $\geq 30$ years of age when they noticed their angiokeratoma.

\section{Duration}

The duration that PEAKERs were present prior to diagnosis was described in 32 men. One patient's lesions were noted after circumcision [9]. Another patient's initial angiokeratoma was congenital; however, the recurrence of his penile angiokeratoma had been present for 6 months prior to diagnosis and treatment [5].

The angiokeratoma duration, for the remaining 30 patients, ranged from 2 months to 30 years (median, 4 years). The PEAKER was

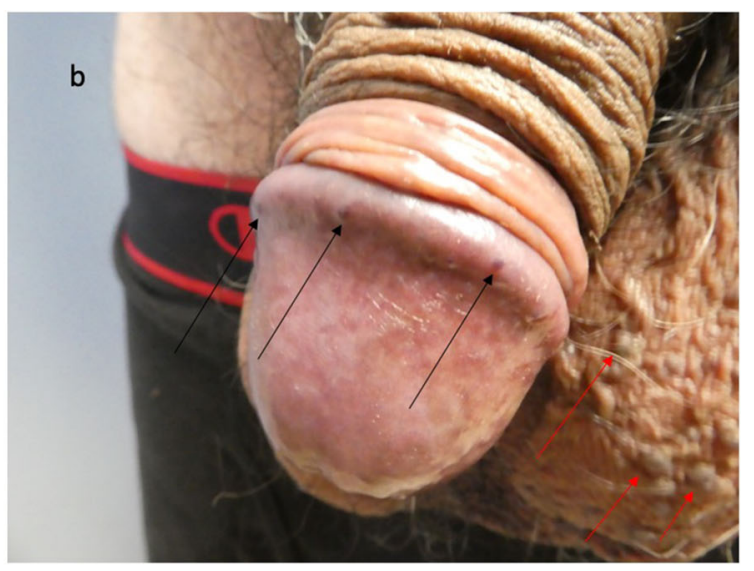

(b) views of the glans penis and the left side of the scrotum showed $6<1 \mathrm{~mm}$ purple papules on the glans penis (corona) (black arrows) and $>253$-mm purple papules on the left side of the scrotum (red arrows); a confluent superficial white plaque with distal peeling was also noted on the glans penis. After the diagnosis of genital angiokeratomas was shared with the patient, he desired no additional treatment for the PEAKERs on the corona of his glans penis. He has been referred for laser treatment of the scrotal angiokeratomas

present $<10$ years for $80 \%$ (24 of 30 ) of the men. Indeed, only $17 \%$ (5 of 30) of the men had lesions for $>20$ years prior to diagnosis.

\section{Symptoms}

Angiokeratoma-associated symptoms in men with PEAKERs were the same as those observed in either male or female patients with genital angiokeratomas. Information was available for 41 of the 54 patients. The PEAKERs were asymptomatic for $61 \%$ (25 of 41 ) of the men $[4,6,9,10,12-15,18-20,22,24]$.

PEAKER-associated symptoms were observed in $39 \%$ (16 of 41 ) of men. These included bleeding (12 men) [5, 7, 8, 16, 23, 25], current report, increasing size of the lesions (10 men) $[5,7,11,16,17,25]$, abrupt onset of new lesions (2 men) [17, 21], pain (2 men) [16], current report, and/or pruritus (2 men) [7], current report. Multiple symptoms were experienced by ten men: two symptoms (eight men) [5, 7, 25], current report, or three symptoms (two men) $[7,16]$. Five of the men with symptomatic 


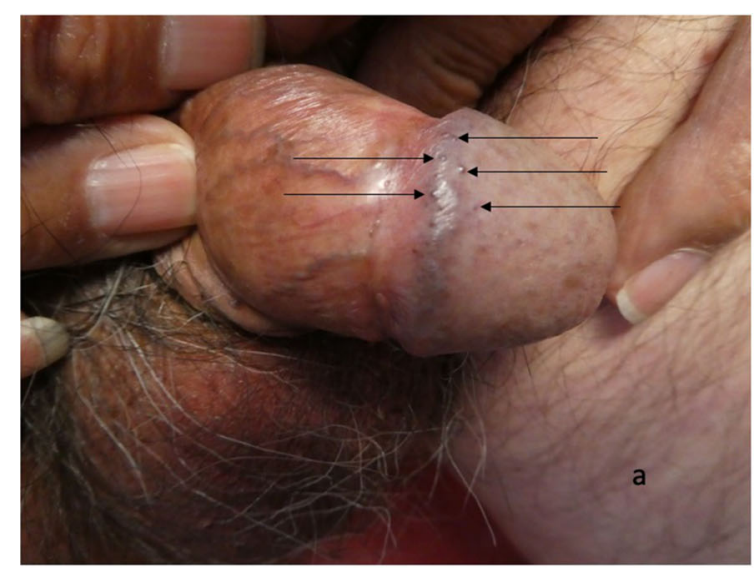

Fig. 3 Penile angiokeratomas (PEAKERs) and scrotal angiokeratomas on the genitalia of a 75-year-old man. His past medical history was significant for herpes simplex virus type 2 infection of his penis. Two years earlier-at age 73 years - he had developed concurrent chancroid and primary syphilis, which were adequately treated with $1 \mathrm{~g}$ of oral azithromycin and 2.4 million units of intramuscular benzathine penicillin, respectively. He presented for evaluation of scrotal erythema of 2-year duration; occasionally, he also experienced pruritus or pain (burning and stinging) or both of his scrotum, penile shaft and/or glans penis. Intermittent symptomatic relief was provided with topical corticosteroid (betamethasone dipropionate 0.05\%)

PEAKERs (bleeding and angiokeratomas that were increasing in size and number) also sought evaluation and treatment since they were experiencing anxiety and social embarrassment [25].

\section{Location}

The location of the PEAKERs were available for all of the 54 men. More than $90 \%$ of the angiokeratomas were on either the glans penis (55.5\%, 30 men) $[4-6,8,9,11-18,20-25]$, current report, or the penile shaft $(35 \%, 19$ men) $[1,7,10,12,13,15,16,23]$. Less commonly, the lesions were on either the foreskin $(5.5 \%$, three men) [1] or both the glans penis and the penile shaft (4\%, two men) [13].

Thirty-seven percent (11 of 30 ) of the men with PEAKERs only on their glans penis had angiokeratomas that were specifically located on the corona $[4,8,11,14,16,18,21,22,25]$,

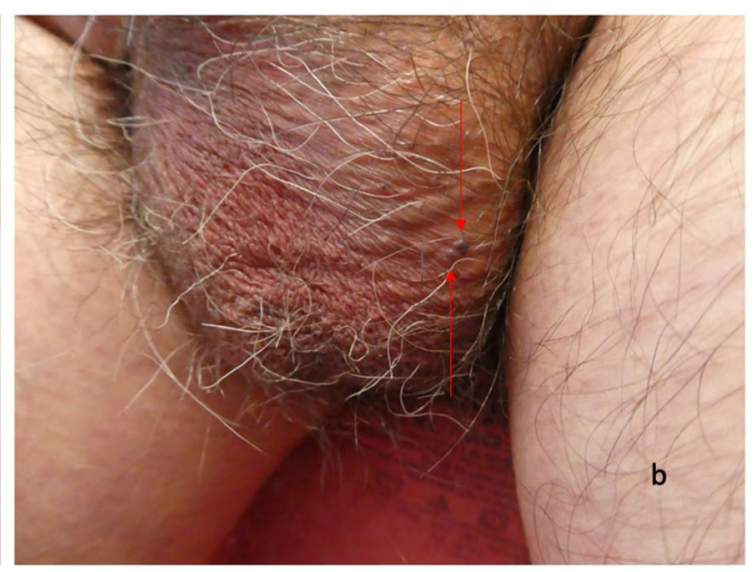

ointment or calcineurin inhibitor (tacrolimus $0.03 \%$ ) ointment. Examination showed 1-mm purple papules on the left side of his penis (black arrows): four on the corona and one on the glans (a); in addition, he had diffuse erythema of his scrotum and two 2-3 $\mathrm{mm}$ purple papules on the left side of his scrotum (red arrows) (b). He had not been aware of the PEAKERs on his glans penis or the angiokeratomas on his scrotum; he was not interested in any therapeutic intervention for his genital angiokeratomas. His genital symptoms completely resolved after he began to treat the affected areas with a lotion containing menthol $(0.5 \%)$ and camphor $(0.5 \%)$

current report. Two of the men with glans penis PEAKERs had an angiokeratoma located on the urethral meatus of their penis [5], current report. Three of the 11 men with coronal PEAKERs also had additional angiokeratomas on other areas of the glans penis $[11,18]$, current report.

Angiokeratomas were also present on the scrotum in 11 of the 54 men (20\%) with PEAKERs [6, 7, 10, 15, 18, 23, 25], current report. This was more commonly observed in men with PEAKERs on their glans penis (seven men) $[6,18,25]$, current report, than those with PEAKERs on their penile shaft (four men) $[7,10,15,23]$.

\section{Clinical Presentation}

The number of angiokeratomas were provided for 47 patients. A solitary PEAKER was described in $32 \%$ (15 or 47$)$ of the men $[1,4,20]$. Two to 
20 angiokeratomas were observed in ten men $[5,13,15,16,19,22,24]$, current report, and > 30 lesions were reported in three men [7, 21], current report. Descriptive terms were used to define the number of lesions for the remaining men: multiple (six men) [8, 9, 14, 17, 18, 23], few to several (five men) [12], few to several dozen (five men) [25], many (two men) $[6,10]$ and numerous (one man) [18] (Table 1).

The size of the angiokeratomas was described for 28 of the men; for 1 of these men, the lesions were described as very small [6]. The smallest lesions were noted in two men: $0.5 \mathrm{~mm}[17,21]$. In 15 men, the PEAKERs ranged from 1-5 mm $[4,8,9,11,12,14,20,24]$, current report. However, an upper size limit between 7-9 mm was noted in three men $[15,16,25]$, and one man's lesions ranged in size from 10-15 mm [23] (Table 1).

The color of PEAKERs was similar to those of non-genital angiokeratomas and non-penile genital angiokeratomas. The angiokeratoma's color was mentioned in 27 men. Purple was the most frequent color (22 men) [4-6, 9, 12, 15, $17,18,20-22,24]$, current report. Other common colors included red (16 men) $[8,9,11,12,15,18,19,21,24]$ and blue $(13$ men) $[8,11,12,15,17,19,21]$. Black (three men) $[14,16,20]$ and tan (one man) [16] angiokeratomas were less often observed (Table 1).

Only 30\% (8 of 27 men) of the PEAKERs were one color $[4-6,14,22]$, current report. The angiokeratomas were two colors in ten of the men: purple and red (four men) $[9,18,24]$, blue and red (three men) $[8,11,19]$ and either black and purple [20], black and tan [16] or blue and purple [17] in one man. Nine men's PEAKERs were blue, purple and red $[12,15,21]$ (Table 1).

\section{Dermoscopy}

Dermoscopy, a noninvasive technique, enhances the visualization of structures within the epidermis, dermoepidermal junction and superficial dermis [45]. Dermoscopic examination can be used, along with clinical findings, to make the diagnosis of a PEAKER $[12,13,24]$. In addition, dermoscopy can be helpful to differentiate angiokeratomas form other cutaneous tumors in the morphologic differential diagnosis such as melanoma and pigmented basal cell carcinoma $[45,46]$.

Similar to the common pathology features of genital and nongenital angiokeratomas, the dermoscopy findings of PEAKERs are also those observed in solitary angiokeratomas. A study of 32 solitary angiokeratomas was performed to describe the dermoscopic features associated with these lesions. At least $50 \%$ of the solitary angiokeratomas had the following six dermoscopic structures (Table 3) [45]: dark lacunae, whitish veil, erythema, peripheral erythema, red lacunae and hemorrhagic crusts. Indeed, the most consistent dermoscopic pattern observed was the concurrent presence of dark lacunae (corresponding to the dilated vascular spaces in the upper dermis) and a whitish veil (corresponding to the hyperkeratosis and acanthosis in the epidermis) [45].

\section{Pathology}

\section{Light Microscopy}

The pathologic changes of PEAKERs are similar to those of non-penile genital angiokeratomas and non-genital angiokeratomas. There is usually hyperkeratosis, without the retention of cell nuclei (orthokeratosis), of the stratum corneum or thickening of the epidermis (acanthosis) or both. Often an 'epidermal collarette'-created by elongation of the epidermal rete ridges at the periphery of the lesion-extends into the underlying dermis and surrounds the dermal vascular component of the angiokeratoma $[1,10,24,47,48]$.

Thin-walled, ectatic, endothelial cell-lined (without cellular atypia or mitoses) vascular spaces are present in the papillary dermis. Some of these blood vessels are thrombosed $[1,10,24,47,48]$.

\section{Electron Microscopy}

Gioglio et al. described the ultrastructural findings observed on electron microscopy of the angiokeratoma endothelial cells of the vessel walls in the papillary dermis; the specimens were from scrotal angiokeratomas from four 
Table 3 Dermoscopic features of penile angiokeratomas (PEAKERs) ${ }^{\mathrm{a}}$

\begin{tabular}{|c|c|c|}
\hline $\begin{array}{l}\text { Dermoscopic } \\
\text { feature }\end{array}$ & AwF & Histopathologic correlation \\
\hline Dark lacunae & $94 \%$ & $\begin{array}{l}\text { This corresponds to the dilated vascular spaces in the upper dermis. The dark violaceous, dark } \\
\text { blue or black colors of the lacunae correspond to vascular spaces that are partially or completely } \\
\text { thrombosed }\end{array}$ \\
\hline Erythema & $69 \%$ & $\begin{array}{l}\text { This appears as pinkish homogeneous areas within the lesion. It corresponds not only to } \\
\text { inflammation of the lesion, but also extravasated erythrocytes in the papillary dermis }\end{array}$ \\
\hline $\begin{array}{l}\text { Hemorrhagic } \\
\text { crusts }\end{array}$ & $53 \%$ & This corresponds to bleeding that occurs in the dermis \\
\hline $\begin{array}{l}\text { Peripheral } \\
\text { erythema }\end{array}$ & $53 \%$ & $\begin{array}{l}\text { This appears as pinkish homogeneous areas at the periphery of the lesion. It corresponds not only } \\
\text { to inflammation of the lesion, but also extravasated erythrocytes in the papillary dermis }\end{array}$ \\
\hline Red lacunae & $53 \%$ & $\begin{array}{l}\text { These are sharply ovoid or round red or red-blue structures. They correspond to wide and dilated } \\
\text { vascular spaces in the upper or middle dermis }\end{array}$ \\
\hline Whitish veil & $91 \%$ & $\begin{array}{l}\text { This appears as an ill-defined structureless area with an overlying whitish ground-glass film. It } \\
\text { corresponds to hyperkeratosis and acanthosis of the epidermis }\end{array}$ \\
\hline
\end{tabular}

$A w F$ angiokeratomas with feature, $\%$ percent observed in solitary angiokeratomas

a These features are based on a multicenter retrospective study of 32 histopathologically proven specimens of solitary angiokeratomas [45]

hospitalized men: three with alcoholic cirrhosis and one with renal cell carcinoma. The investigators not only noted that endothelial cell cytoplasmic organelles (such as endoplasmic reticulum and mitochondria) were scarce, but also observed vacuoles with scarcely electronopaque content in the endothelial cell cytoplasm. The researchers postulated that both the presence of the endothelial cell vacuoles and decreased quantity of cytoplasmic organelles resulted from the vascular dilation and subsequent stasis of the angiokeratoma vessels [48].

\section{Diagnosis}

The diagnosis of a PEAKER may be suspected based upon the clinical evaluation of the vascular lesion during an examination of the glans penis, penile shaft or both. Indeed, in some of the men, angiokeratomas may also be present on the scrotum. Hence, in the 54 men with PEAKERs, the diagnosis of angiokeratoma was established based on the morphologic appearance of the lesion in 32\% (17 of 54) of the men
$[4,7,10,15,16,18,22,25]$, current report. For the remainder of the patients, the PEAKERs were diagnosed either from microscopic evaluation of a skin biopsy from a lesion $(48 \%, 26$ of 54 men) $[1,5,6,8,9,11,14,17-21,23,24]$ or using dermoscopy (20\%, 11 of 54 men) [12, 13]. The high percent of biopsy-confirmed diagnoses of PEAKERs results from half (13 of 26) of the men with biopsied angiokeratomas originating from a single retrospective study in which PEAKER patients were obtained from pathology files of individuals with tissue biopsy specimens diagnosed as angiokeratomas [1].

\section{Differential Diagnosis}

\section{Clinical}

Imperial and Helwig's study, which included genital and non-genital angiokeratomas, discovered that the correct diagnosis by the clinician was only made in 3\% (3 of 116) of the submitted specimens. Pigmented lesions, such as nevus (35\%, 41 of 116 specimens) and melanoma (15\%, 17 of 116 specimens), were often 
the suspected diagnosis. These diagnoses were based on clinical history and symptoms of the lesions-a solitary lesion of long-standing duration that had suddenly become larger and darker and bled intermittently [1]. In addition, in the retrospective study by Naranjo Sintes et al., $20 \%$ of the angiokeratomas were clinically diagnosed as melanomas [41].

Genital angiokeratomas-particularly of the scrotum and vulva-can also mimic recurrent cancer. Scrotal angiokeratomas have masqueraded as recurrent squamous cell carcinoma of the penis in men following penectomy $[49,50]$. Also, in women, vulvar angiokeratomas have mimicked recurrent squamous cell carcinoma of the cervix following hysterectomy and radiotherapy [37].

Similar to non-genital angiokeratomas, not only other melanocytic lesions (such as the spindle and epithelioid cell nevus of Spitz) but also other benign tumors are in the clinical differential diagnosis of PEAKERs. These include epithelial lesions (such as prurigo nodularis and seborrheic keratosis), fibrous lesions (such as dermatofibroma), infectious lesions (such as condyloma accuminata, condyloma lata, molluscum contagiosum and verruca vulgaris) and vascular lesions (such as capillary aneurysm, hemangioma, lymphangioma and pyogenic granuloma). In addition, malignant epithelial tumors (such as basal cell carcinoma and squamous cell carcinoma) and vascular neoplasms (such as angiosarcoma and Kaposi sarcoma) can mimic angiokeratomas [3, 10, 13, 14, 16, 47].

PEAKERs have rarely been described to mimic keratoacanthomas. The giant angiokeratomas on the penile shaft of a 72-year-old man clinically masqueraded as keratoacanthomas by appearing as dome-shaped nodules with a keratinous plug in the central crateriform ulcer. In addition, the man had multiple smaller angiokeratomas presenting as keratotic papules on both the shaft of his penis and his scrotum [23].

\section{Pathologic}

The pathologic differential diagnosis of PEAKERs includes other vascular lesions such as a capillary aneurysm (which is also referred to as a capillary varix), cherry angioma (which is also referred to as a senile angioma), hemangioma, lymphangioma and pyogenic granuloma. Capillary aneurysm, cherry angioma and hemangioma typically do not show reactive acanthosis and hyperkeratosis. Lymphangiomas contain proteinaceous material in the vessels; however, they can be difficult to differentiate from angiokeratomas if erythrocytes have spilled into the vascular spaces. Pyogenic granulomas, similar to angiokeratoma, often have an 'epidermal collarette' surrounding the vascular component of the lesion; however, the mixed inflammatory infiltrate typically present in pyogenic granuloma is usually absent in non-inflamed angiokeratomas [1].

\section{Associated Conditions}

Investigators have postulated that the development of scrotal angiokeratomas more readily occurs in individuals who have conditions that may be associated with either long-standing venous hypertension or a prolonged increase in venous pressure $[10,47]$. Some of these conditions were observed in men with PEAKERs. They included hemorrhoids (two men) [6, 18], hydrocele (one man) [18], inguinal hernia (one man) [6], prostate hypertrophy (two men) $[18,21]$, radiotherapy for prostate cancer (one man) [24] and varicocele (two men) [10, 18].

Two of the men with unilateral PEAKERs had ipsilateral conditions $[10,18]$; whether this is a coincidental observation that only occurred in these two individuals or there is a bona fide increased occurrence of angiokeratoma-associated conditions developing on the side to which the PEAKERs are located remains to be determined. Both of the men had a condition that was associated with elevated venous pressure-a varicocele [10, 18]. Also each man had another condition on the same side as their angiokeratoma: a renal cyst [18] or a chronic dermatitis [10].

The first man was 16 years old when he noticed his first PEAKER on his left penile shaft. During the next 5 years, several additional angiokeratomas appeared only on the left side of the shaft of his penis and the left side of his scrotum. The investigators who described his 
angiokeratomas attributed them to the varicosities and chronic dermatitis on the left side of his scrotum [10].

The second man was 66 years old. He had multiple PEAKERs located on the left side of his glans penis and his scrotum. His medical history was significant for irritable bowel syndrome, a left-sided renal cyst and left varicocele [18].

Lichen sclerosis et atrophicus (also referred to as balanitis xerotica obliterans) can present as white glistening patches on men's glans penis or foreskin or both. It was described by the investigators as a comorbidity in a 41-year-old man whose PEAKERs on his glans penis and penile shaft had been present for 8 years. The images included in the report of a 71-year-old man-who had PEAKERs on his glans penis that had been present for 5 years-also showed clinical features suggestive of lichen sclerosus et atrophicus; his angiokeratomas appeared 5 months after he completed radiation therapy to treat an adenocarcinoma of his prostate that had been diagnosed 1 year earlier [24].

One of the patients described in this reportthe 75-year-old man who had PEAKERs not only on the corona but also other areas of his glans penis-also had scrotal angiokeratomas and a red scrotum. Scrotal erythema has previously been observed in men with angiokeratomas of the scrotum. Indeed, one group of researchers noted that approximately 50\% of their patients with genital angiokeratomas had diffuse redness of their scrotum [51].

\section{Workup}

A PEAKER is a benign vascular lesion. It can be solitary or multiple. The angiokeratomas can only occur on the penis or the scrotum. When a PEAKER is discovered, the patient's skin should be examined for additional angiokeratomas on the rest of his body. If there are only genital angiokeratomas, no additional workup is necessary. However, if angiokeratomas are diffusely present, evaluation for a lysosomal storage disease (such as Fabry's disease) and possible referral to a medical geneticist should be considered.

\section{Pathogenesis}

Several hypotheses have been proposed for the pathogenesis of angiokeratomas. They are not necessarily mutually exclusive. However, the primary etiology of angiokeratomas not associated with inherited metabolic disorders is different from that associated with lysosomal storage diseases.

Angiokeratoma corpus diffusum is one of the dermatologic hallmarks of Fabry disease (which is also known as Anderson-Fabry disease). The angiokeratoma typically appear in boys between the ages of 5 and 10 years. They are not only often found in the bathing trunk area (including the penis, scrotum and buttocks), but also many other sites: axilla, ears, feet, hands, inner thighs, lower back, mucosa (of the digestive, respiratory and genitourinary tracts) and umbilicus [26-30].

However, in addition to Fabry disease, angiokeratoma corpus diffusum can also be observed in individuals with other lysosomal storage diseases. These include aspartylglucosaminuria, fucosidosis, galactosialidosis, betagalactosidosis, beta-mannosidosis and Schindler/Kanzaki disease. In addition, albeit rare, familial or sporadic angiokeratoma corpus diffusum has been observed in individuals without an enzymatic defect-associated metabolic disease $[47,52]$.

The mechanism of angiokeratoma pathogenesis is similar for individuals with a lysosomal storage disease. For example, in patients with Fabry disease, deficiency of the lysosomal enzyme alpha-galactosidase $A$ results in the accumulation of glycosphingolipid globotriaosylceramide in the lysosomes of cutaneous endothelial cells. This eventually causes the capillary walls to weaken and secondary ectasia, resulting in the development of angiokeratomas, to occur [26-30].

The end-stage vessel changes in the pathogenesis of angiokeratomas in PEAKERs are indeed similar to those postulated for the development of angiokeratomas in patients with lysosomal storage diseases: capillary dilation and subsequent vascular ectasia. However, the mechanism that results in dilation of the capillaries is different. In patients with genital 
angiokeratomas, trauma of chronic irritation causes an alteration in either the blood vessel or the dermal stroma surrounding the blood vessel or both; subsequently, the capillaries dilate and there is impaired contractibility of the vessel wall that eventually is accompanied by vascular ectasia and angiokeratoma development $[1,10,13,34,43,47,53]$.

Alternatively, stasis can result in venous back pressure and hypertension, which causes the vessels to dilate. Eventually the over distension of the vessel causes elastic tissue fragmentation and permanent damage to the vessel wall. Thereafter, vascular ectasia occurs and angiokeratomas develop [1, 10, 13, 34, 43, 47, 53].

Some of the patients with PEAKERs have associated conditions that can cause and/or occur secondary to localized venous hypertension: hemorrhoids, hydroceles, inguinal hernia, prostate hypertrophy and varicoceles $[6,10,18,21]$. Also, genital angiokeratomas have been observed in men and women following radiation therapy to the area [24, 37, 49]. Scrotal angiokeratomas have developed in men following surgery in the adjacent area-penectomy for squamous cell carcinoma of the penis $[49,50]$.

In summary, the primary event in the development of PEAKERs-similar to other genital and non-genital angiokeratomas-is the vascular ectasia of the vessels in the papillary dermis. The changes in the overlying epidermis-acanthosis, hyperkeratosis and elongation of the rete ridges into the dermis to surround the vascular component-subsequently occur as a secondary response to the vascular ectasia in the underlying dermis $[1,47]$.

\section{Treatment}

The management of angiokeratoma may include observation or interventional modalities. Cryotherapy, electrosurgery, excision, laser surgery and sclerotherapy have all been successfully used to treat angiokeratomas. Indeed, several of these techniques have been used in men with PEAKERs [54-56].

The treatment of PEAKERs was described in 41 men; the retrospective study of 13 men, based on pathology files, did not provide any information on the management of their angiokeratomas [1]. Twenty-seven percent (11 of 41 men) had no additional treatment of the penile angiokeratomas $[4,6,9,11,17,18,21,22]$, current report. Palliative management with emollients was recommended for a 71-year-old man whose clinicians decided to avoid any physical treatments [24].

Lasers were used to treat $56 \%$ ( 23 or 41 men) of the PEAKERs. Several of these men were treated with a pulsed dye laser: 595-nm variable pulsed (six men) [13], $585 \mathrm{~nm}$ (five men) [25] or $595 \mathrm{~nm}$ (one man whose congenital angiokeratoma-at the same location 7 years earlierhad been surgically removed during infancy) [5]. Neodymium-doped: yttrium aluminum garnet laser was also successfully utilized: 1064 nm (four men) [7, 15] or $532 \mathrm{~nm}$ (one man) [8]. One of the individuals who received four therapy sessions with the 1064-nm neodymiumdoped: yttrium aluminum garnet laser, a 43-year-old man whose penile shaft and scrotal angiokeratomas had been present for 13 years, developed atrophic scars at the sites of treatment [15].

One group of investigators performed a single-center, randomized, comparative, observerblinded study that included five men with PEAKERs; they compared treatment of the angiokeratomas with a 595-nm pulsed dye laser on one side and 1064-nm long-pulse neodymium-doped: yttrium aluminum garnet laser on the other side in each of the individuals. Both lasers were effective and safe in the treatment of the angiokeratomas. However, the overall mean improvement for neodymiumdoped: yttrium aluminum garnet laser-treated angiokeratomas was statistically superior compared with the lesions treated with the pulsed dye laser [12].

A 52-year-old man had multiple, verrucoussurfaced angiokeratomas on the corona of his glans penis. His PEAKERs were successfully treated with three cycles of combined laser therapy. A 2940-nm erbium: yttrium aluminum garnet laser was initially used to remove the epidermal hyperkeratosis followed by a 532-nm potassium-titanyl-phosphate laser to treat the 
dermal vascular component of the angiokeratoma [19].

Four men (10\%), including one of our patients, had PEAKERs that were treated with electrocautery $[10,14,23]$, current report. Radiofrequency was used to excise multiple giant PEAKERs on the penile shaft that mimicked keratoacanthomas in a 72-year-old man who also had several smaller angiokeratomas on his penis and scrotum that were treated with electrocautery [23]. One of the men had a massive recurrence of his angiokeratoma following electrocautery treatment [10]; however, there was no recurrence of the angiokeratoma either 8 months [current report] or 1 year [23] after electrocautery treatment for the other men.

One man's PEAKER was successfully treated without recurrence by surgically excising his angiokeratoma; however, prior to surgery, cryotherapy with liquid nitrogen had failed to resolve the lesion [20]. Another man's PEAKER required three sequential excisions since it recurred twice after the initial and second surgeries [16]. A third patient had his congenital penile angiokeratoma excised during infancy; however, it recurred at the same site when he was 7 years of age; it was successfully treated without recurrence (at 1 year follow-up) using a 595-nm pulsed dye laser after three treatment sessions [5].

\section{Recurrence}

Only 30 of the 41 men (73\%) with PEAKERs had their angiokeratomas treated. Ten percent (3 of these 30 men) had either a recurrent (two men) $[5,16]$ or a persistent (one man) [20] angiokeratoma. The persistent PEAKER was an angiokeratoma on the glans penis of a 58-year-old man that remained after initial treatment with cryotherapy using liquid nitrogen; the lesion was completely removed-and did not recurfollowing an excisional biopsy [20].

One patient with a recurrent PEAKER was a 7-year-old boy with a congenital angiokeratoma on the glans penis involving the meatus. The PEAKER had been surgically removed during infancy and subsequently appeared in the same location. The recurrent angiokeratoma was completely removed after three treatment sessions using a 595-nm pulsed dye laser [5].

The second patient with a recurrent PEAKER was a 34-year-old man whose penile shaft angiokeratoma was associated with two recurrences after appropriate surgical excisions. A third surgical procedure, using an elliptical incision and including subcutaneous tissue, was performed. The surgical margins were examined and found to be negative for angiokeratoma [16].

\section{CONCLUSION}

PEAKERs are a variant of genital angiokeratomas in men. This subtype of angiokeratoma of Fordyce is more commonly observed than CLANKERs-its embryologic analog (clitoral angiokeratoma) in women. To date, PEAKERs have been described in 54 men. PEAKERs typically appear in younger men; only half of the men were $\geq 30$ years of age when they noticed their penile angiokeratoma. However, the mean duration of time before evaluation was 4 years, ranging from 2 months to $>30$ years. Symptoms were only present in $39 \%$ of the men. Bleeding and increasing size were the most common symptoms; less frequent symptoms were abrupt onset of angiokeratomas, pain and pruritus. PEAKERs were most commonly located on either the glans penis $(55.5 \%)$ or the penile shaft (35\%); less often, they were on the foreskin $(5.5 \%)$ or both the glans penis and penile shaft $(4 \%)$. Thirty-seven percent of patients with PEAKERs on the glans penis had lesions located only on the corona; 3 of these 11 men with PEAKERs on their corona also had angiokeratoma on other areas of the glans penis. Two of the men with glans penis PEAKERs had an angiokeratoma at the urethral meatus. In addition, $20 \%$ of patients with PEAKERs also had angiokeratomas on their scrotum. The number of angiokeratomas ranged from 1 to $>30 ; 32 \%$ of the men only had a solitary PEAKER. The size of the angiokeratoma ranged from $0.05-15 \mathrm{~mm}$; however, most of the PEAKERs were between 1-5 $\mathrm{mm}$. Seventy percent of the PEAKERs were more than one color; the most 
common colors were purple, red and blue. The diagnosis of the PEAKER was confirmed by either biopsy (48\%) or dermoscopy (20\%); however, clinical features established the diagnosis for the remaining $32 \%$ of the patients. The PEAKERs were not treated for $27 \%$ of the men. Laser therapy was the most common modality used for $56 \%$ of the men; these included: pulsed dye laser, neodymium-doped: yttrium aluminum garnet laser, and combined laser treatment with erbium: yttrium aluminum garnet laser and potassium-titanyl-phosphate laser. Less common interventions included electrocautery, radiofrequency, excision, unsuccessful treatment with cryotherapy using liquid nitrogen and palliative management with emollients. PEAKER recurrence or persistence following treatment was observed in $10 \%$ of the men: two men after excision and one man after cryotherapy, respectively.

\section{ACKNOWLEDGEMENTS}

We thank the participants of the study.

Funding. No funding or sponsorship was received for this study or publication of this article.

Authorship. All named authors meet the International Committee of Medical Journal Editors (ICMJE) criteria for authorship of this manuscript, take responsibility for the integrity of the work as a whole, and have given final approval of the version to be published. The opinions expressed in the manuscript are those of the authors.

Disclosures. Nicholas Celano has nothing to disclose. Philip R. Cohen is a member of the journal's Editorial Board.

Compliance with Ethics Guidelines. This article is based on previously conducted studies and does not contain studies with human participants or animals performed by any of the authors. However, the patients or their parent signed a consent form providing permission for including the clinical photographs in this article.

Data Availability. Data sharing is not applicable to this article as no data sets were generated or analyzed during the current study.

Open Access. This article is licensed under a Creative Commons Attribution-NonCommercial 4.0 International License, which permits any non-commercial use, sharing, adaptation, distribution and reproduction in any medium or format, as long as you give appropriate credit to the original author(s) and the source, provide a link to the Creative Commons licence, and indicate if changes were made. The images or other third party material in this article are included in the article's Creative Commons licence, unless indicated otherwise in a credit line to the material. If material is not included in the article's Creative Commons licence and your intended use is not permitted by statutory regulation or exceeds the permitted use, you will need to obtain permission directly from the copyright holder. To view a copy of this licence, visit http:// creativecommons.org/licenses/by-nc/4.0/.

\section{REFERENCES}

1. Imperial R, Helwig EB. Angiokeratoma. A clinicopathological study. Arch Dermatol. 1967;95(2): 166-75.

2. Fordyce JA. Angiokeratoma of the scrotum. J Cutan Genitourin Dis. 1896;14:81-7.

3. Cohen PR, Young AW Jr, Tovell HM. Angiokeratoma of the vulva: diagnosis and review of the literature. Obstet Gynecol Surv. 1989;44(5):339-46.

4. Basu P, Cohen PR. Penile angiokeratoma (Peaker): a distinctive subtype of genital angiokeratoma. Cureus. 2018;10(12):e3793.

5. Burnett CT, Kouba DJ. A rare case of congenital angiokeratoma of the glans penis treated using a 595-nm pulsed dye laser. Dermatol Surg. 2012;38(12):2028-30.

6. Bruce DH. Angiokeratoma circumscriptum and angiokeratoma scroti. Arch Dermatol. 1960;81(3): 388-93. 
7. Zeng Y, Zhan K, Xie WL, Lin QZ. Angiokeratoma of Fordyce response to long pulsed Nd:YAG laser treatment. Dermatol Ther. 2016;29(1):48-51.

8. Bechara FG, Huesmann M, Stucker M, Altmeyer P, Jansen T. An exceptional localization of angiokeratoma of Fordyce on the glans penis. Dermatology. 2002;205(2):187-8.

9. Carrasco L, Izquierdo MJ, Farina MC, Martin L, Moreno C, Requena L. Strawberry glans penis: a rare manifestation of angiokeratomas involving the glans penis. Br J Dermatol. 2000;142(6):1256-7.

10. Imperial R, Helwig EB. Angiokeratoma of the scdrotunm (Fordyce type). J Urol. 1967;98(3): 379-87.

11. Masuria BL, Gupta LK, Batra A, Kothiwala RK, Singh MK. Angiokeratoma of Fordyce on unusual site. Indian J Dermatol Venereol Leprol. 1999;65(5): 240-1.

12. Ibrahim SM. Pulsed dye laser versus long pulsed Nd: YAG laser in the treatment of angiokeratoma of Fordyce: a randomized, comparative, observerblinded study. J Dermatolog Treat. 2016;27(3): 270-4.

13. Baumgartner J, Simaljakova M. Genital angiokeratomas of Fordyce 595-nm variable-pulsed dye laser treatment. J Cosmet Laser Ther. 2017;19(8):459-64.

14. Dudani S, Maskara R. Multiple angiokeratomas of Fordyce: lesions at an unusual site causing a diagnostic dilemma. Indian J Dermatopathol Diagn Dermatol. 2014;1(1):46-7.

15. Ozdemir M, Baysal I, Engin B, Ozdemir S. Treatment of angiokeratoma of Fordyce with longpulsed neodymium-doped yttrium aluminum garnet laser. Dermatol Surg. 2009;35(1):92-7.

16. Pianezza ML, Singh D, Van der Kwast T, Jarvi K. Rare case of recurrent angiokeratoma of Fordyce on penile shaft. Urology. 2006;68(4):891.e1-3.

17. Keen MA, Hassan I. Eruptive angiokeratomas on the glans penis. Indian J Dermatol. 2014;59(4):424.

18. Erkek E, Basar MM, Bagci Y, Karaduman A, Bilen $\mathrm{CY}$, Gokoz A. Fordyce angiokeratomas as clues to local venous hypertension. Arch Dermatol. 2005;141(10):1325-6.

19. Bechara FG, Jansen T, Wilmert M, Altmeyer P, Hoffmann K. Angiokeratoma Fordyce of the glans penis: combined treatment with erbium: YAG and $532 \mathrm{~nm}$ KTP (frequency doubled neodymium: YAG) laser. J Dermatol. 2004;31(11):943-5.
20. Beutler BD, Cohen PR. Angiokeratoma of the glans penis. Skinmed. 2017;15(5):343-7.

21. Feramisco JD, Fournier JB, Zedek DC, Venna SS. Eruptive angiokeratomas on the glans penis. Dermatol Online J. 2009;15(10):14.

22. Schleicher S. Derm Dx: violaceous papules on the genitalia. Clinical Advisor. 2019, June 6. https:// www.clinicaladvisor.com/slideshow/derm-x/derm$\mathrm{dx}$-violaceous-papules-on-the-genitalia/.

23. Ghosh SK, Ghosh S, Agarwal M. Multiple giant angiokeratoma of Fordyce on the shaft of the penis masquerading as keratoacanthoma. An Bras Dermatol. 2015;90(3 Suppl 1):150-2. https://doi.org/ 10.1590/abd1806-4841.20153876.

24. Leis-Dosil VM, Alijo-Serrano F, Aviles-Izquierdo JA, Lazaro-Ochaita P, Lecona-Echeverria M. Angiokeratoma of the glans penis: clinical, histopathological and dermoscopic correlation. Dermatol Online J. 2007;13(2):19.

25. Lapidoth M, Ad-El D, David M, Azaria R. Treatment of angiokeratoma of Fordyce with pulsed dye laser. Dermatol Surg. 2006;32(9):1147-50.

26. Larralde $M$, Boggio $P$, Amartino $H$, Chamoles $N$. Fabry disease: a study of 6 hemizygous men and 5 heterozygous women with emphasis on dermatologic manifestations. Arch Dermatol. 2004;140(12): 1440-6.

27. Mohrenschlager M, Henkel V, Ring J. Fabry disease: more than angiokeratomas. Arch Dermatol. 2004;140(12):1526-8.

28. Chan B, Adam DN. A review of Fabry disease. Skin Therapy Lett. 2018;23(2):4-6.

29. Hsu TR, Niu DM. Fabry disease: review and experience during newborn screening. Trends Cardiovasc Med. 2018;28(4):274-81.

30. Bokhari SRA, Zulfigar H, Hariz A. Fabry disease. StatPearls [Internet]. In: StatPearls [Internet]. StatPearls Publishing, Treasure Island, FL; 2020

31. Miller M, Corey GR, Neelon FA. Angiokeratoma of Fordyce. NC Med J. 1990;51(2):71.

32. Thambiah AS, Rajam RV. A report on two benign vascular tumours in dermatology: (I) granuloma pyogenicum and (II) hyperkeratotic haemangioma or keratoangioma. Indian J Vener Dis. 1954;20:94.

33. Imperial R, Helwig EB. Angiokeratoma of the vulva. Obstet Gynecol. 1967;29(3):307-12.

34. Sadowsky LM, Socik A, Burnes A, Rhodes AR. Genital angiokeratomas in adult men and women: 
prevalence and predisposing factors. J Cutan Med Surg. 2019;23(5):513-8.

35. Fogagnolo L, Cintra ML, Velho PE. Angiokeratoma of the vulva. An Bras Deramtol. 2011;86(2):333-5.

36. Bogan F, Bucak IH. Rare giant angiokeratoma of the vulva: a case report. Balkan Med J. 2017;34(2): $163-4$.

37. Haidopoulous DA, Rodolakis AJ, Elsheikh AH, Papaspirou I, Diakomanolis E. Vulvar angiokeratoma following radical hysterectomy and radiotherapy. Acta Obstet Gynecol Scand. 2002;81(5): 466-7.

38. McNeely TB. Angiokeratoma of the clitoris. Arch Pathol Lab Med. 1992;116(8):880-1.

39. Yigiter M, Arda IS, Tosun E, Celik M, Hicsonmez A. Angiokeratoma of the clitoris: a rare lesion in an adolescent girl. Urology. 2008;71(4):604-6.

40. Yamazaki $M$, Hiruma $M$, Irie $H$, Ishibashi A. Angiokeratoma of the clitoris: a subtype of angiokeratoma vulvae. J Dermatol. 1992;19(9): 553-6.

41. Naranjo Sintes R, Pereda Hernandez J, Delgado Florencio V, Linares Solano J. Angiokeratoma. Apropos of 93 cases. Med Cutan Ibero Lat Am. 1988;16(3):255-61.

42. Izaki M. Angiokeratoma of the scrotum (Fordyce). Keio J Med. 1952;1(1):62-8.

43. Bean WB. Vascular spiders and related lesions of the skin. Springfield: Charles C. Thomas; 1958. p. 262-264.

44. Patrizi A, Neri I, Trevisi P, Landi C, Bardazzi F. Congenital angiokeratoma of Fordyce. J Eur Acad Dermatol Venereol. 1998;10(2):195-6.

45. Zaballos P, Daufi C, Puig S, Argenziano G, MorenoRamirez D, Cabo H, Marghoob AA, Llambrich A, Zalaudek I, Malvehy J. Dermoscopy of solitary angiokeratomas: a morphological study. Arch Dermatol. 2007;143(3):318-25.
46. Sahin MT, Turel-Ermertcan A, Ozturkcan S, Turkdogna P. Thrombosed solitary angiokeratoma of Mibelli simulating malignant melanoma: the importance of dermoscopy in differential diagnosis. J Eur Acad Dermatol Venereol. 2006;20(1):102-4.

47. Schiller PI, Itin PH. Angiokeratomas: an update. Dermatology. 1996;193(4):275-82.

48. Gioglio L, Porta C, Moroni M, Nastasi G, Gangarossa I. Scrotal angiokeratoma (Fordyce): histopathological and ultrastructural findings. Histol Histopathol. 1992;7(1):47-55.

49. Malalasekera AP, Goddard JC, Terry TR. Angiokeratoma of Fordyce simulating recurrent penile cancer. Urology. 2007;69(3):576.e13-14.

50. Bisceglia M, Carosi I, Castelvetere M, Murgo R. Multiple Fordyce-type angiokeratomas of the scrotum. An iatrogenic case. Pathologica. 1998;90(1): 46-50.

51. Miller C, James WD. Angiokeratoma of Fordyce as a cause of red scrotum. Cutis. 2002;69(1):50-1.

52. Kanitakis J, Allombert C, Doebelin B, Deroo-Berger MC, Grande S, Blanc S, Claudy A. J Cutan Pathol. 2005;32(7):506-11.

53. Majumdar S, Saha SS. Angiokeratoma of scrotum (Fordyce). Indian J Dermatol Venereol Leprol. 1996;62(1):52-4.

54. Cohen PR. A case of scrotal rejuvenation: laser treatment of angiokeratomas of the scrotum. Dermatol Ther (Heidelb). 2019;9(1):185-92.

55. Nguyen J, Chapman LW, Korta DZ, Zachary CB. Laser treatment of cutaneous angiokeratomas: a systematic review. Dermatol Ther. 2017;30(6): e12558.

56. Riahi RR, Cohen PR. Genital rejuvenation of scrotal and vulvar angiokeratomas: laser therapy of scrotum and vulva lesions. Skinmed, in press. 